\title{
Galactic mass-losing AGB stars probed with the IRTS. I.
}

\author{
T. Le Bertre ${ }^{1}$, M. Matsuura ${ }^{2,3,4}$, J. M. Winters ${ }^{1,5}$, H. Murakami $^{2}$, I. Yamamura $^{2}$, \\ M. Freund ${ }^{2,6}$, and M. Tanaka ${ }^{2,7}$ \\ 1 DEMIRM, UMR 8540, Observatoire de Paris, 61 Av. de l'Observatoire, 75014 Paris, France \\ 2 Institute of Space and Astronautical Science, 3-1-1 Yoshinodai, Sagamihara, Kanagawa 229-8510, Japan \\ 3 Department of Astronomy, University of Tokyo, 7-3-1 Hongo, Bunkyo, Tokyo 113-0033, Japan \\ 4 UMIST, Department of Physics, PO Box 88, Manchester M60 1QD, UK \\ 5 Max-Planck-Institut für Radioastronomie, Auf dem Hügel 69, 53121 Bonn, Germany \\ 6 Infrared Astrophysics Branch, Code 685, Goddard Space Flight Center, Greenbelt, MD 20771, USA \\ 7 Department of Physics, Catholic University of America, Washington, DC 20064, USA
}

Received 25 April 2001 / Accepted 12 July 2001

\begin{abstract}
AGB mass-losing sources are easy to identify and to characterize in the near-infrared range $(1-5 \mu \mathrm{m})$. We make use of the near-infrared data acquired by the Japanese space experiment IRTS to study a sample of sources detected in the 2 celestial strips surveyed by the IRTS. Mass-loss rates and distances are estimated for 40 carbon-rich sources and 86 oxygen-rich sources of which 8 are probably of S-type. Although the sample is small, one sees a dependence of the relative contribution of the two kinds of sources to the replenishment of the interstellar medium (ISM) on the galactocentric distance. E.g. from 6 to $8 \mathrm{kpc}$, oxygen-rich sources in our sample contribute 10-12 times as much as carbon rich sources, whereas from 10 to $12 \mathrm{kpc}$, the latters contribute $3-4$ times as much as the formers. Therefore, one would expect a gradient in the composition of the ISM between 6 and $12 \mathrm{kpc}$ from the Galactic Centre, especially in its dust component. Most of the replenishment (>50\%) by AGB stars is due to sources with mass-loss rate larger than $10^{-6} M_{\odot} \mathrm{yr}^{-1}$.
\end{abstract}

Key words. stars: carbon - stars: mass-loss - stars: AGB and post-AGB - ISM: evolution - galaxy: solar neighbourhood - infrared: stars

\section{Introduction}

Red giants at some epochs during their evolution along the Asymptotic Giant Branch (AGB) lose matter at a large rate. By this process, these stars contribute to more than $50 \%$ of the replenishment of the Interstellar Medium (ISM). In the Solar Neighborhood this proportion might even be higher (Sedlmayr 1994). Furthermore, due to convective mixing, their external layers are enriched in material nuclearly processed in the interiors. Therefore during these epochs of intense mass loss they also participate in the chemical evolution of stellar systems. In particular, it is considered that AGB stars are responsible for the carbon enrichment of the ISM and are the major source for the injection of dust grains (Gehrz 1989).

There are basically 2 kinds of mass-losing AGB stars, those with an oxygen-rich composition $(\mathrm{C} / \mathrm{O}<1)$ and those with a carbon-rich one $(\mathrm{C} / \mathrm{O}>1)$. These 2 kinds correspond to a different level of chemical enrichment in the atmosphere. The difference appears clearly in the molecular bands and in the dust features that they display in their spectra, particularly in the infrared range. Our

Send offprint requests to: T. Le Bertre, e-mail: Thibaut.LeBertre@obspm.fr objective is to quantify the contribution of both types of mass losing AGB sources to the cosmic cycle of matter. The spectral range from 1 to $10 \mu \mathrm{m}$ is crucial to perform this work (Epchtein 1999).

With such objective in mind, we want to exploit the data obtained in the near-infrared range $(1-4 \mu \mathrm{m})$ by the space experiment IRTS. In this first paper we examine in detail a subset of recently released data which covers a small sample $(\sim 120)$ of mass-losing AGB sources. We will concentrate on the description of the method that we apply to the IRTS data in order to derive mass-loss rates. An important feature of our method is to be sensitive to the present mass loss of AGB stars. In a second paper (II), when the full data set is made available, we will apply the same approach to a larger sample $(\sim 1000)$ with the objective of estimating the mass injection rate from the 2 kinds of AGB stars in the Solar Neighborhood.

\section{Presentation of the data}

\subsection{The IRTS mission}

The IRTS (Infrared Telescope in Space) is a $15 \mathrm{~cm} \mathrm{He-}$ cooled telescope which has been operated in space during 38 days in March and April 1995 (Murakami et al. 1996). 
It surveyed in a scanning mode two strips in the sky amounting to a total of 2700 square degrees (or $7 \%$ of the sky). The first one (or North-scan) was inclined at $15^{\circ}$ over the Galactic Plane (GP) and crossed it at $l^{\mathrm{II}} \approx 0^{\circ}$ and $180^{\circ}$; it covered $\sim 2 \%$ of the sky. The second one (or South-scan) was inclined at $50^{\circ}$ over the GP and crossed it at $l^{\mathrm{II}} \approx 49^{\circ}$ and $229^{\circ}$; it covered $\sim 5 \%$ of the sky. The main purpose of the IRTS mission was to perform a survey of the infrared $(1-1000 \mu \mathrm{m})$ diffuse emission with moderate spatial resolution (Okuda et al. 1997).

The telescope was equipped with four focal instruments of which two are of relevance also for the study of point sources: the NIRS (Near-Infrared Spectrometer) and the MIRS (Mid-Infrared Spectrometer). Both are grating spectrometers with $8^{\prime} \times 8^{\prime}$ entrance apertures. The NIRS was fitted with two linear arrays of $12 \mathrm{InSb}$ detectors covering the wavelength ranges $1.38-2.51 \mu \mathrm{m}$ and $2.85-$ $3.96 \mu \mathrm{m}$. The spectral resolution is $\sim 0.1 \mu \mathrm{m}$ for point sources (and $0.13 \mu \mathrm{m}$ for diffuse sources) and allows to see clearly molecular bands in late-type stars such as those of $\mathrm{CO}$, at 1.5 and $2.3 \mu \mathrm{m}, \mathrm{H}_{2} \mathrm{O}$, at $1.4,1.9$ and $2.7 \mu \mathrm{m}$ and $\mathrm{C}_{2} \mathrm{H}_{2}-\mathrm{HCN}$, at $3.1 \mu \mathrm{m}$, (Yamamura et al. 1997; Matsuura et al. 1999). The MIRS covers the wavelength range $4.5^{-}$ $11.7 \mu \mathrm{m}$ with spectral resolution $\sim 0.3 \mu \mathrm{m}$. It mainly detected the Unidentified Infrared Bands (UIB) at 6.2, 7.7, 8.6 and $11.3 \mu \mathrm{m}$ in the diffuse interstellar medium (Onaka et al. 1996), and silicate and silicon-carbide dust features in late-type stars, as well as molecular bands.

In January 1996, the satellite was recovered from space by the Space Shuttle allowing for post-flight inspection and re-calibration (Murakami et al. 2001; Onaka et al. 2001).

\subsection{The NIRS data}

The basis of the NIRS data processing is described in Noda et al. (1996). Events (i.e. blocks of data where the signals from the detectors are significantly higher than the background) are extracted from each scan after identification and removal of glitches, and background subtraction. The final version of the data processing pipeline is still under development. The pointing was reconstructed at IPAC using records by a focal-plane star sensor operating at $1.25 \mu \mathrm{m}$ and by gyros (Moshir et al. 1997). In general, the accuracy of the source coordinates is $\sim 1^{\prime}$ for in-scan direction and $\sim 2^{\prime}$ for cross-scan direction. However some scans are found to be affected by systematic effects which are larger. Also for some individual events the position could be uncertain by as much as the aperture size $\left(\sim 8^{\prime}\right)$ along the cross-scan direction. On the basis of positions the events are cross-identified, and eventually recombined into sources.

Spectra are measured for each event. The data were corrected using post-flight measurements and a raytracing simulation of the instrument (Murakami et al. 2001). The absolute wavelength calibration of the instrument is excellent. However, for point sources, depending

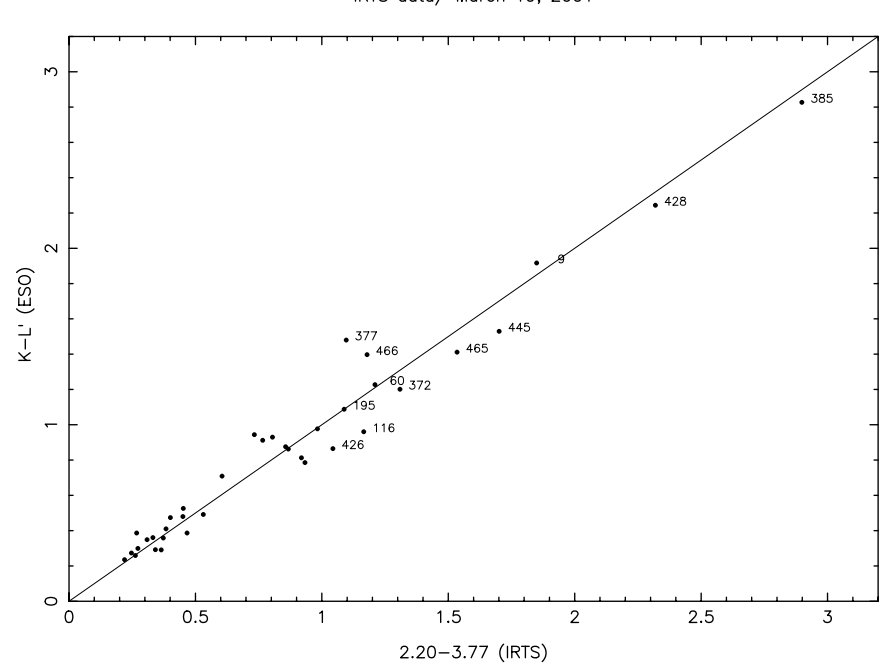

Fig. 1. $[2.20-3.77]_{\mathrm{IRTS}}$ versus $\left(K-L^{\prime}\right)_{\mathrm{ESO}}$ for 37 NIRS sources. The reddest sources are labelled with an index referencing every NIRS source.

on the centering in the aperture, the spectra moved over the array by $\pm 0.05 \mu \mathrm{m}$. The photometric calibration was derived from the infrared calibration of four non-variable late-type giants ( $\theta$ Her, $\theta$ Cnc, $\kappa$ Gru, IRC +60231), which have been well observed by the NIRS (Cohen et al. 1999). The photometric stability of the NIRS system has been checked to be better than $5 \%$ over the whole mission (including the orbits crossing the South Atlantic Anomaly, SAA, where the instruments were exposed to showers of high-energy protons).

The comparison of the magnitude scale of the IRTS with ground-based photometry is not straightforward because many detected sources are variable and because the data set presently available is small. Also the fluxes measured for a point source depend on its exact positioning in the aperture and therefore also on its spectral shape. However one finds good one-to-one correlations between the NIRS fluxes and the fluxes obtained from the ground in $K$ and $L^{\prime}$ although with some scatter $( \pm 0.3 \mathrm{mag})$. Color indices are less sensitive to variability than magnitudes. In Fig. 1, we compare the [2.20-3.77] index from the IRTS to $K-L^{\prime}$ for sources belonging to the sample described in the next section, and also observed by us at ESO (Fouqué et al. 1992; Le Bertre 1992, 1993). This plot is consistent with a one-to-one relation between the 2 color indices.

For some scans the telemetry was operated at a rate of $3 \mathrm{kbps}$ (kilo-bit per second) instead of $6 \mathrm{kbps}$. In these situations (which affected about $12 \%$ of the sources, the majority of which belongs to the North-scan) only the fluxes from every alternate (odd-numbered) channels were transmitted, which means that the $2.20 \mu \mathrm{m}$ fluxes (channel 16) are not available. In Fig. 2 we present the [2.20-2.30] color (obtained from channels 16 and 15) versus the [2.30-3.77] color (obtained from channels 15 and 3 ) for sources which have been observed at $6 \mathrm{kbps}$. The dispersion is large, but one notes first a negative trend, then from $\sim(0.4,-0.1)$ a 
IRTS data/ March 19, 2001

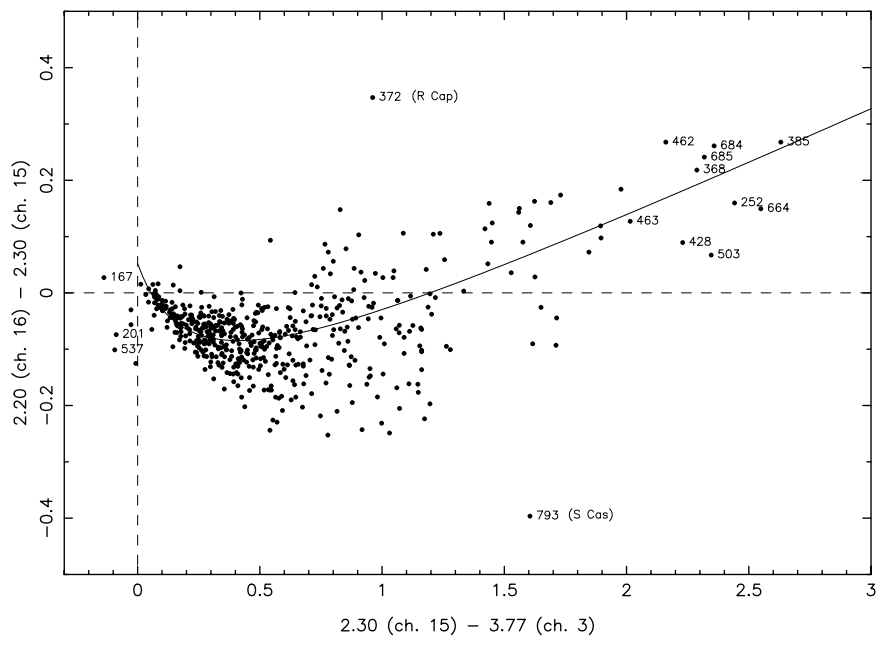

Fig. 2. $[2.20-2.30]$ versus [2.30-3.77] for 531 NIRS sources. $\mathrm{S}$ Cas is an S star with a deep CO absorption band and R Cap, a carbon star (see text).

positive one. This dependence can be understood as the effect of an increasing $2.3 \mu \mathrm{m}$ CO absorption band for stars of late spectral type which tends to be filled in by dust emission for those which undergo mass loss. A color-color relation can be derived empirically:

$[2.20-2.30]=0.2[2.30-3.77]-0.30+\frac{0.09}{[2.30-3.77]+0.25}(1)$

for $[2.30-3.77] \geq 0.08$.

It is worth noting that the scatter depends on the $[2.30-$ 3.77 color index. It is increasing from $[2.30-3.77]=0.0$ to $\sim 0.5$, then is maximum up to $[2.30-3.77] \sim 1.0$, and slightly decreasing beyond. The outlier object No. 793 (S Cas) is an S star which shows a very deep absorption at $2.3 \mu \mathrm{m}$. The NIRS spectrum of the carbon star R Cap is exceptional in that it shows $\mathrm{CO}$ in emission at $2.3 \mu \mathrm{m}$. It could be an artefact. However, it is worth noting that some carbon Miras have been observed to show molecular emission $\left(\mathrm{C}_{2}\right)$ in the optical range during major fading events (Lloyd-Evans 1997). Also in the oxygen-rich Mira $\mathrm{T}$ Cep, $\mathrm{SO}_{2}$ has been seen in emission at several epochs by ISO (Yamamura et al. 1999). Finally, Lançon \& Wood (2000) found CO in emission at $2.3 \mu \mathrm{m}$ in the spectrum of $\mathrm{UEqu}$; but the nature of this star is unclear.

Carbon stars tend to fall above the line and oxygenrich stars, below. This effect could be due to the contribution of $\mathrm{H}_{2} \mathrm{O}$ absorption at $2.3 \mu \mathrm{m}$ in oxygen-rich stars. However, the 2 populations overlap widely; also $\mathrm{S}$ Cas, an S star, shows the largest absorption at $2.3 \mu \mathrm{m}$. Another source of intrinsic scatter might be the variability. Indeed Miras are known to show deeper molecular absorption bands than Semi-Regular variables and nonvariable giants of the same effective temperature, because of the pulsation-induced extension of their outer atmospheric layers. Also, the depths of their molecular bands are strongly variable with phase (Yamamura \& de Jong 2000; Matsuura et al. 2001).

\subsection{Description of the sample}

About 1000 sources have been detected by the MIRS and an estimated 10000 , by the NIRS. The MIRS processing is almost completed and a first catalogue containing $\sim 700$ spectra is in preparation (Yamamura et al. 2001). For many sources, the MIRS spectra were affected by a varying response of the detectors developing after the satellite crossed the South Atlantic Anomaly (SAA). The current MIRS process rejects about $5 \%$ of the MIRS events due to the SAA. The remaining events show drops of the responsivity up to $\sim 10 \%$ (Onaka et al. 2001). Fortunately, this effect was always much smaller for the NIRS detectors $(\leq 5 \%)$ so that in general we could ignore the effect of the SAA on the NIRS events. On the other hand, the NIRS data are more affected by confusion and by the dependence of the wavelength calibration on the source position in the aperture.

From this set of 1000 sources detected by the MIRS we have first removed about 200 items which are Solar System objects (asteroid Ceres and space debris), extended sources or compact (as compared to the $8^{\prime}$ beamsize) H II regions. The latters are easily identified by their UIBs which stand out in the $6-11 \mu \mathrm{m}$ range. Counterparts have been searched for the remaining 800 sources, which therefore are expected to be stellar, in the NIRS database. Plausible identifications have been obtained for 598 sources. The stellar MIRS sources which have no NIRS counterpart are extremely red sources, which were too faint in the near-infrared range to be detected, or sources which have been lost. Two kinds of sources have been lost, (i) sources which saturated the NIRS detectors, because they are too bright in the near-infrared, (ii) sources which could not be properly extracted due to confusion (e.g. close to the Galactic Plane).

We base our present analysis on this sub-set of 598 sources of which 531 were observed at $6 \mathrm{kbps}$ and the remaining 67 , at $3 \mathrm{kbps}$. The processing of the complete NIRS data is under progress and the catalogue with all NIRS point sources will be released in the future. It is expected to contain 10-15 times as many sources as the current sub-set. The latter should be on average fainter and/or bluer than those of the present sample. The limiting magnitudes for the detection of a NIRS point source are $\sim 8.0$ at [2.20], and $\sim 7.0$ at [3.77], but with some degradation close to the Galactic Plane due to confusion.

\subsection{Discussion}

For most of the sources we could identify an IRAS counterpart. In Fig. 3 we present the color diagram IRAS [12-25] versus IRTS [2.20-3.77] for the corresponding 554 sources. Again some sources (44) have been lost, mainly because of astrometric inaccuracies which 
IRTS data/ March 19, 2001

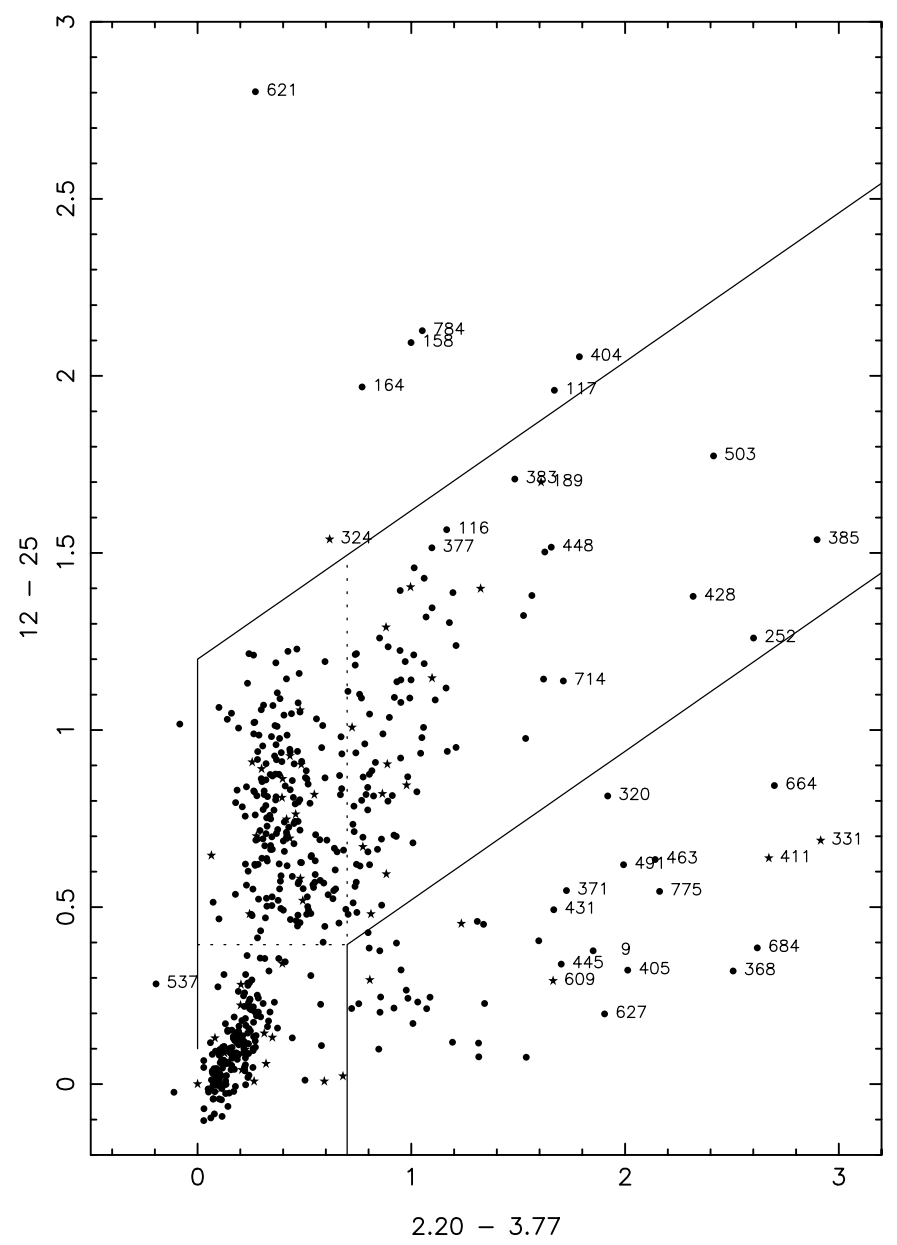

Fig. 3. IRAS [12-25] versus [2.20-3.77] for the 554 NIRS sources cross-identified with IRAS; $(\bullet)$ sources observed at $6 \mathrm{kbps},(\star)$ sources observed at $3 \mathrm{kbps}$.

prevented an unambiguous identification with IRAS. On the other hand, due to the large entrance aperture used for the observations $\left(8^{\prime} \times 8^{\prime}\right)$, some sources might have been incorrectly identified to an IRAS source especially close to the Galactic Plane. For the sources observed with $3 \mathrm{kbps}$ the $2.20 \mu \mathrm{m}$ flux was estimated from the $2.30 \mu \mathrm{m}$ one using relation 1 . As this approach presents some uncertainties, in the following we will keep these sources separated from the others. This diagram resembles similar ones (i.e. [12-25] versus $K-L^{\prime}$ ) already published (see e.g. Epchtein et al. 1987, Fig. 5). Two clumps are noted: the first one around $[2.20-3.77] \sim 0.2,[12-25] \sim 0.1$, and a second one around $[2.20-3.77] \sim 0.3,[12-25] \sim 0.8$.

The first clump (三Valinhos "a" in Epchtein et al. 1987) corresponds to sources, mainly late-type giants, with photospheric colors. The second clump (Valinhos "b") corresponds to red-giant stars with weak circumstellar dust shell emission. Sources with mass-loss rate $\sim 10^{-8} M_{\odot} \mathrm{yr}^{-1}$ are expected to be found in this area. Le Bertre et al. (1994) note that this group contains also stars with cool shells (i.e. sources which have recently undergone a drastic reduction in their rate of mass loss). These stars may still show up with a large value of the index [12-25]. In general, in an IRAS colors diagram (van der Veen \& Habing 1988) they would be found in the regions VIa and VIb. To the right of $[2.20-3.77]=0.7$, one finds stars which are presently undergoing mass loss at a large rate (Valinhos "o2", "o3" and "c"). Most of those are red giants, but a few supergiants can be found here and also possibly some young stars. Furthermore, beyond this limit the data points split into 2 bands which are discussed below (Sect. 3.3).

Finally one notes a few sources with peculiar colors. Sources with $[2.20-3.70] \leq 0.0$ and a large $[12-25](\geq 0.25)$ index can be the products of the merging of two closeby but unrelated objects. The same applies to sources above the line $[12-25]=0.42 \times[2.20-3.70]+1.2$. However it should be noted that some real objects (e.g. post-AGB sources) can also be found in these regions. The source labelled 621(三 IRAS 05198+3325) has been associated to a young stellar object and the source 404(三 IRAS 062451013), to a T Tauri star.

The proportions of the different classes of sources in the Epchtein et al. (1987) sample and in the present one are expected to be different because the former were extracted from a survey at $2 \mu \mathrm{m}$ and the latter from the MIRS survey (i.e. at $\sim 8 \mu \mathrm{m}$ ).

\subsection{AGB mass-losing stars}

We will restrict our analysis of the mass-losing AGB stars to the sources with $[2.20-3.77] \geq 0.7$ and $[12-25]<0.42 \times$ $[2.20-3.77]+1.2$. Therefore, in our final sample we keep all objects presently undergoing mass loss at a large rate $\left(\geq 3 \times 10^{-8} M_{\odot} \mathrm{yr}^{-1}\right)$. The sources with a lower mass-loss rate are excluded and their contribution will have to be evaluated separately. We also exclude the stars which in the past had a large $\dot{M}$, but since have experienced an important reduction of this rate.

\section{Exploitation of the data}

\subsection{Bolometric magnitude}

We determine the bolometric magnitude, $m_{\mathrm{bol}}$, for each source by applying a bolometric correction, $B C_{2.20}$, to the [2.20] magnitude:

$m_{\mathrm{bol}}=[2.20]+B C_{2.20}$.

This correction is estimated from the $[2.20-3.77]$ index by:

$B C_{2.20}=2.7$,

for $[2.20-3.77] \leq 2.1$, and

$B C_{2.20}=6.1-1.62 \times[2.20-3.77]$,

for $[2.20-3.77] \geq 2.1$.

The justification for these relations is given in the Appendix. 


\subsection{Distance and interstellar extinction correction}

The average luminosity of a mass-losing AGB star is expected to be $\sim 8000 L_{\odot}$. We adopt an absolute bolometric magnitude $M_{\mathrm{bol}}=-5.01$. Then, the distance of each source is estimated consistently after correction of [2.20] and [2.20-3.77] for interstellar extinction. These estimates should be handled with care. The sources are variable and $m_{\text {bol }}$ may change by 1 mag between minimum and maximum. Also, the true average luminosity may differ from $8000 L_{\odot}$ by a factor up to 2 . Therefore the estimates of the distances to individual sources are uncertain by a factor 1.8 .

The interstellar extinction along a line of sight is determined from an analytical description of the distribution of dust in the Solar Neighborhood (Jones et al. 1981). In this approach the local absorption is an exponential function of $r$, the distance to the Galactic Centre, and $z$, the distance to the Galactic Plane. At the Sun position, the local absorption at $2.20 \mu \mathrm{m}, A_{2.20}^{*}$, is taken to be $0.07 \mathrm{mag} \mathrm{kpc}^{-1}$, and at $3.77 \mu \mathrm{m}, A_{3.77}^{*}, 0.02 \mathrm{mag} \mathrm{kpc}^{-1}$. The scale height, in the $Z$ direction, is taken to be $0.1 \mathrm{kpc}$, and the scale length, in the $R$ direction, $4 \mathrm{kpc}$. Finally we adopt a distance of the Sun to the Galactic Centre of $8.5 \mathrm{kpc}$ and assume that the Sun is in the Galactic Plane. We do not consider the effects of the spiral arms and of the Molecular Ring. This description of the interstellar extinction in the Solar Neighborhood is consistent with the more recent one of Wainscoat et al. (1992), except that we do not apply truncation.

In general the corrections for interstellar extinction are small $(<0.15 \mathrm{mag}$ at $2.20 \mu \mathrm{m})$. The effect on the estimated distance is at most $7 \%$, which is negligible considering the other sources of uncertainty. This is not only because the extinction in the IR is small but also because the corrections on [2.20] and [2.20-3.77] have opposite effects on $m_{\text {bol }}$ which tend to cancel for the reddest sources $([2.20-3.77]>2.1)$.

The main effect is to move a few sources (10) from just above $[2.20-3.77]=0.7$ to just below. For consistency, these sources have been removed from the final sample. However, as the distances are uncertain by a factor 1.8, the interstellar extinction might have been overestimated for some of them. It means that individually they could meet the criterion $[2.20-3.77]_{\text {dered. }} \geq 0.7$. Their names and properties are reported in Table 1.

We find that in our sample most of the sources should be within $\sim 3.5 \mathrm{kpc}$ from the Sun, but some sources are found up to $6.5 \mathrm{kpc}$. In the $Z$ direction, we get sources up to $\sim 1.5 \mathrm{kpc}$ from the GP.

\subsection{Spectral classification}

The NIRS data covers the wavelength range 1.4-4.0 $\mu \mathrm{m}$ which contains various molecular bands such as those of $\mathrm{H}_{2} \mathrm{O}(1.9 \mu \mathrm{m})$ or $\mathrm{C}_{2} \mathrm{H}_{2}+\mathrm{HCN}(3.1 \mu \mathrm{m})$. These features in principle allow us to separate O-rich from C-rich sources
Table 1. Properties of the sources with $[2.20-3.77] \geq 0.7$ and removed from the analysis. The source No. 510 has been observed at $3 \mathrm{kbps}$.

\begin{tabular}{rcccc}
\hline Index & {$[2.20]$} & {$[2.20-3.77]$} & {$[12-25]$} & IRAS name \\
\hline 82 & 3.53 & 0.70 & 1.11 & $15455-6108$ \\
180 & 4.15 & 0.79 & 0.82 & $16538-4633$ \\
192 & 3.27 & 0.74 & 1.21 & $16575-4344$ \\
213 & 3.30 & 0.74 & 1.18 & $17116-4036$ \\
289 & 3.29 & 0.73 & 0.73 & $17372-2632$ \\
469 & 3.50 & 0.70 & 0.48 & $18141+0340$ \\
551 & 3.63 & 0.75 & 0.23 & $19122+1830$ \\
499 & 3.23 & 0.72 & 0.21 & $19155+0847$ \\
524 & 3.44 & 0.76 & 1.10 & $19271+1354$ \\
510 & 2.31 & 0.72 & 1.01 & $19248+1122$ \\
\hline
\end{tabular}

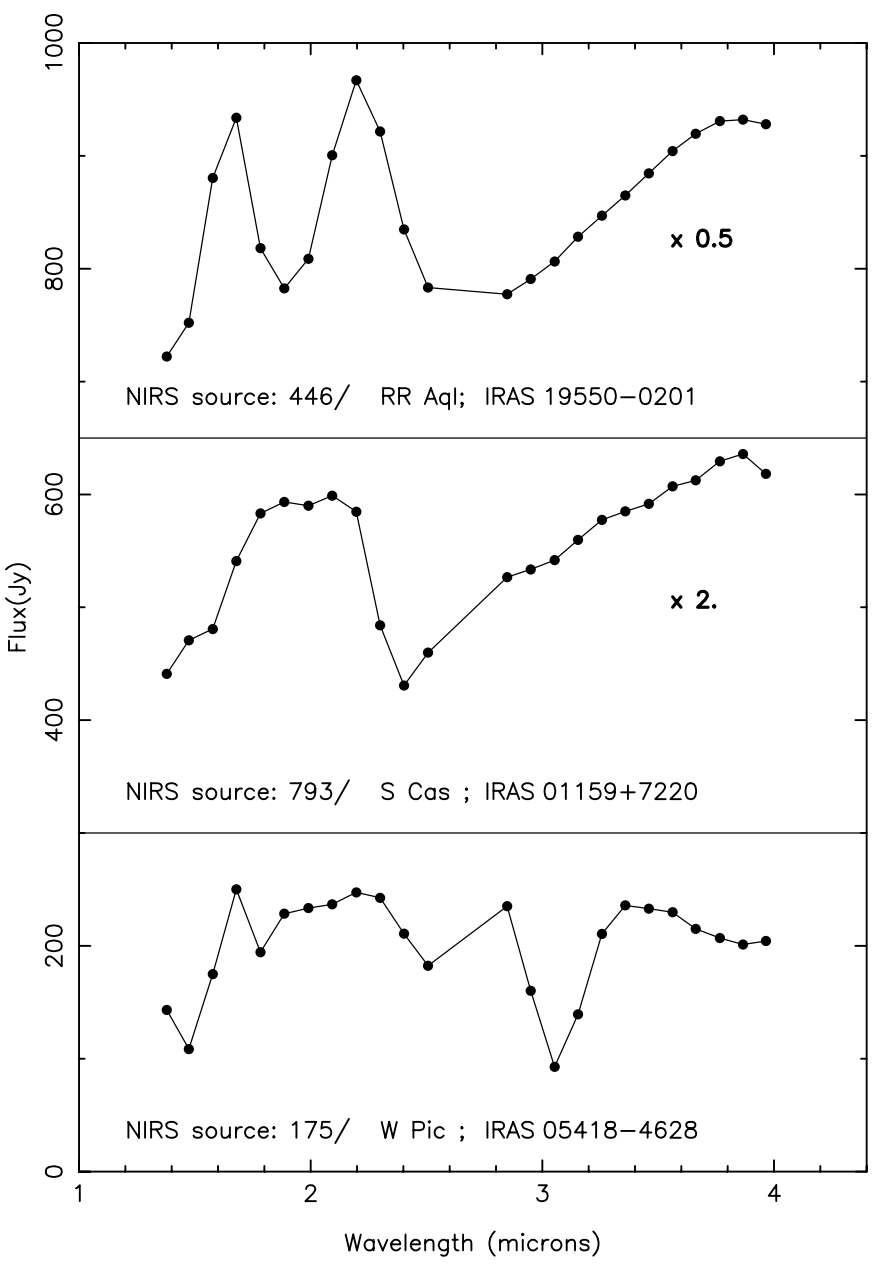

Fig. 4. Typical NIRS/IRTS spectra of mass-losing AGB sources. From bottom to top: W Pic, a carbon star; S Cas, an S-type star and RR Aql, an M-type star. Note the deep $\mathrm{H}_{2} \mathrm{O}$ band at $1.9 \mu \mathrm{m}$ in RR Aql and the $\mathrm{C}_{2} \mathrm{H}_{2}+\mathrm{HCN}$ band at $3.1 \mu \mathrm{m}$ in W Pic.

(Fig. 4). However, in the case of very red sources these bands are partially filled in by dust emission.

A method based on the color indices, $K-L^{\prime}$ and IRAS [12-25], has been developped by Epchtein et al. (1987). In the corresponding color-color diagram, C-rich 
and O-rich sources surrounded by circumstellar shells separate clearly. The reason for this separation lies in the very different optical properties of the carbon-rich and oxygen-rich dust grains in the infrared range. In Fig. 5 we present the $[12-25]$ versus [2.20-3.77] colors diagram for the NIRS sources of our sample which satisfy the criteria $[2.20-3.77] \geq 0.7$ and $[12-25] \leq 0.42 \times[2.20-3.77]+1.2$. O-rich and C-rich sources separate clearly. We adopt the same dividing line as in Epchtein et al.:

$[12-25]=0.42 \times[2.20-3.77]+0.10$,

for $[2.20-3.77] \geq 0.7$.

Each of the 130 NIRS spectra has been checked individually. In general this inspection confirms the validity of the Epchtein et al. (1987) classification criterion: the $\mathrm{H}_{2} \mathrm{O}$ band at $1.9 \mu \mathrm{m}$ is found in sources belonging to the "Orich" region (Fig. 4; RR Aql) and the band at $3.1 \mu \mathrm{m}$, in sources belonging to the "C-rich" region (Fig. 4; W Pic).

For 8 sources (Table 2) belonging to the "O-rich" region, none of these bands can be seen. However, they show clearly a deep CO absorption at $2.3 \mu \mathrm{m}$ (Fig. 4; S Cas). When available the IRAS Low Resolution Spectrum confirms the O-rich composition of the circumstellar shell and 3 sources are known to be of S-type (Nos. 793, 207, and 686). We consider that the 5 other sources which have similar NIRS spectra are likely to be also of S-type. We note however that some stars which are identified to be of S-type (e.g. TTCMa) do show the $\mathrm{H}_{2} \mathrm{O}$ absorption band at $1.9 \mu \mathrm{m}$. It is known that $\mathrm{S}$ stars exhibit a range of spectroscopic properties intermediate between those of M stars and those of pure S stars. Piccirillo (1980) shows that $\mathrm{H}_{2} \mathrm{O}$ tends to disappear from the photospheric layers of S-type stars when the $\mathrm{C} / \mathrm{O}$ abundance ratio becomes greater than 0.9 . The absence of the $1.9 \mu \mathrm{m} \mathrm{H}_{2} \mathrm{O}$ band in our spectra would then point to $\mathrm{S}$ stars with a rather large $\mathrm{C} / \mathrm{O}$ ratio. Finally, $\mathrm{H}_{2} \mathrm{O}$ is found to be weak or even absent in red supergiants spectra (Alvarez et al. 2000), a combined effect of their low surface gravity and higher effective temperature. In this context we note that the source No. 251 might in fact be associated to an M0Ia star (Massey et al. 2001).

Three sources (Nos. 316, 252 and 339) are obviously mis-classified. The 316 NIRS spectrum shows clearly the absorption feature at $3.1 \mu \mathrm{m}$ and the source is associated to GM CMa, a carbon star. Finally 3 sources are unrelated to the AGB (Nos. 709, 714 and 385). Their NIRS spectra are featureless. The sources 709 and 385 are associated to emission line stars of spectral type B and 714 , to an RV Tauri type star. Therefore we have removed the sources 709, 714, 385 and 316 from the sample and corrected the spectral classification of the sources 252 and 339. We thus get a sample of 126 AGB stars of which 40 are carbon-rich and 86, oxygen-rich.

\subsection{Mass-loss rate}

Several methods are used to derive the mass-loss rates of AGB stars from observations. An introduction to this
Table 2. Possible and confirmed mass-losing S-type stars.

\begin{tabular}{rccc}
\hline Index & IRAS name & optical id. & IRAS LRS \\
\hline 793 & $01159+7220$ & S Cas & 22 \\
484 & $05591+0638$ & & \\
168 & $16538-4652$ & & 14 \\
207 & $16598-4117$ & CSS952 & 24 \\
234 & $17187-3750$ & & 26 \\
230 & $17244-3827$ & & 14 \\
251 & $17328-3327$ & & 29 \\
686 & $19354+5005$ & R Cyg & 22 \\
\hline
\end{tabular}

Table 3. Misclassified and contaminating sources. The source No. 339 has been observed at 3 kbps.

\begin{tabular}{rccc}
\hline Index & IRAS name & optical id. & IRAS LRS \\
\hline 709 & $04156+5552$ & CI Cam & \\
714 & $04166+5719$ & TW Cam & \\
385 & $06259-1301$ & HD 45677 & 24 \\
316 & $06391-2213$ & GM CMa & 23 \\
252 & $17209-3318$ & & 43 \\
339 & $17540-1919$ & VVSgr & 28 \\
\hline
\end{tabular}

topic can be found in Lafon \& Berruyer (1991). Up to now, mainly 2 methods have been applied. The first one is based on the modelling of CO rotational lines (Knapp \& Morris 1985) and the second one, on the modelling of the continuum emission observed in the infrared (Jones \& Merrill 1976). In both cases, the modelling assumes that the mass-loss rate has been constant over time, $\sim$ a few thousand years in the first case, a few hundred years in the second. Both methods assume a conversion ratio, from a minor species (CO or dust) to the total mass, which might not be constant. Nevertheless, in general, the two methods agree to within a factor 3 .

However, the mass-loss phenomenon is known to exhibit variations on several timescales. This effect explains that, in fact, different methods may give very different results. For instance, using $\mathrm{CO}$ rotational line data Loup et al. (1993) find $\dot{M} \sim 10^{-5} M_{\odot} \mathrm{yr}^{-1}$ for $\mathrm{R} \mathrm{Scl}$, whereas Le Bertre (1997), using IR continuum data, finds $\sim 10^{-7} M_{\odot} \mathrm{yr}^{-1}$, a factor about one hundred less. The explanation lies in a drastic reduction of the $\mathrm{R} \mathrm{Scl}$ mass loss about 100 years ago, an effect discovered by RowanRobinson et al. (1986) and which can be inferred from the IRAS data which shows an excess at $\lambda \geq 25 \mu \mathrm{m}$. The situation is even more complex if one considers the multiple molecular winds recently brought to evidence from composite CO line profiles (Kahane \& Jura 1996; Knapp et al. 1998). In these cases, the mass-loss rate variations can be uncovered because the properties of the winds, in particular the outflow velocities, are very different. One may suspect that, in other situations where the changes are less radical, such effects would not be detected, but still affect the determination of $\dot{M}$. This is particularly true for the $\mathrm{CO}$ method which probes a shell of typically 


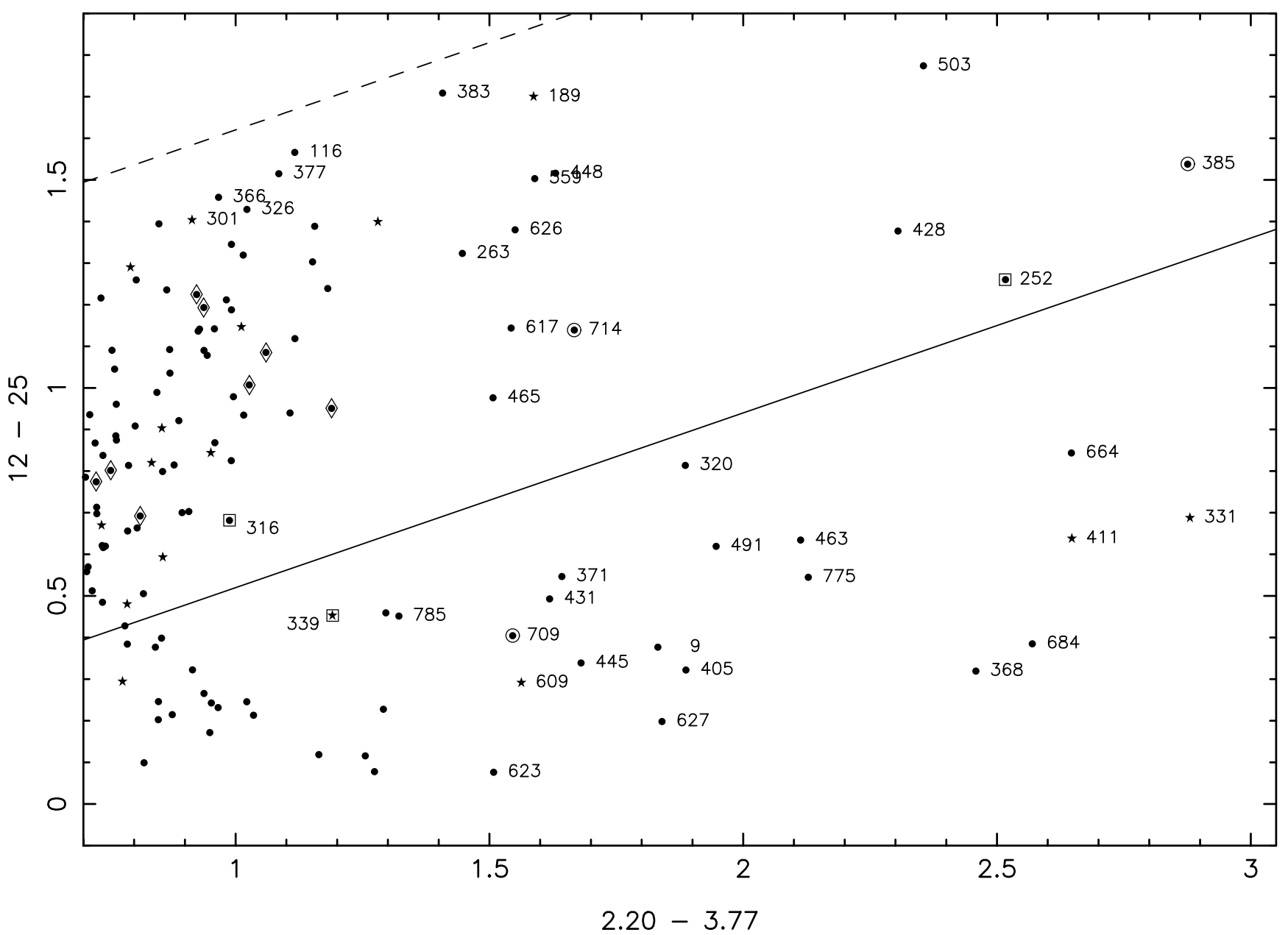

Fig. 5. IRAS [12-25] versus [2.20-3.77] for the selection of 130 mass-losing AGB candidates. The [2.20-3.77] index is corrected for interstellar extinction assuming that each candidate has a luminosity of $8000 \mathrm{~L} \odot$. The solid line separates O-rich (above) from C-rich (below) sources. The reddest sources are labelled with their NIRS indices. $(\bullet)$ sources observed at $6 \mathrm{kbps}$, $(\star)$ sources observed at $3 \mathrm{kbps}$. The diamonds enclose the representative points of S-type stars (Table 2), the squares those of mis-classified sources and the circles those of contaminating sources (Table 3).

$10^{17} \mathrm{~cm}$ in size, which is filled in on a timescale of 1 to a few $10^{3}$ years. The IR method is less sensitive to this problem because it is the most internal parts of the circumstellar dust shells which dominate the IR continuum emission. The near-infrared $(1-5 \mu \mathrm{m})$ continuum emission originates in a shell of $\sim 10^{15} \mathrm{~cm}$, whereas the far-infrared emission $(\lambda \geq 25 \mu \mathrm{m})$ comes from a larger region of $\sim 10^{16} \mathrm{~cm}$ (e.g. Rowan-Robinson et al. 1986). The IR method is thus expected to give an estimate of $\dot{M}$ which fits more closely the present characteristics of the central star than the $\mathrm{CO}$ method.

Both methods can be used to derive mass-loss rates only for well observed sources at known distances from the Sun. To handle large samples, relations allowing to estimate easily $\dot{M}$ have been proposed. For instance Jura (1987) has proposed to estimate $\dot{M}$ from the $60 \mu \mathrm{m}$ IRAS fluxes. This approach works well for sources which have not undergone a recent variation of their mass-loss rate like R Scl. Here also the distances must be known. More recently, Le Bertre (1997) and Le Bertre \& Winters (1998) have shown that the mass-loss rates of AGB stars are correlated with the $K-L^{\prime}$ color index. Their empirical relations are derived from a sample of well studied sources in the Solar Neighborhood for which $\dot{M}$ has been determined through the IR method.

On the basis of these works, we adopt: $\log \dot{M}_{-6}=-9.0 /([2.20-3.77]+1.4)+2.75$,

for C-rich sources, and

$\log \dot{M}_{-6}=-2.75 /[2.20-3.77]+2.25$,

for O-rich sources, where $\dot{M}_{-6}$ is in $10^{-6} M_{\odot} \mathrm{yr}^{-1}$ and [2.20-3.77] has been corrected for interstellar extinction.

In a systematic study, Winters et al. (2000) showed that self-consistent hydrodynamical models which produce winds with $\dot{M} \geq 3 \times 10^{-7} M_{\odot} \mathrm{yr}^{-1}$ follow such kind of relations. They show that these relations hold for various dust-to-gas ratios due to the interplay between massloss rate, dust-to-gas ratio and expansion velocity. In these models, the flux of matter is variable over a timescale of one stellar pulsation period, or a few in the cases of multiperiodic models (Fleischer et al. 1995). Winters et al. 
define the mass-loss rate as a flux average over 20 stellar periods, or $\sim 20-50$ years. On the basis of their study it does not seem meaningful to define a mass-loss rate over a shorter timescale; it is only on such a timescale that AGB sources can be considered as stationary systems. Furthermore, there are evidences that on longer timescales (>40 years) some AGB sources might undergo large variations of the mass loss (Mauron \& Huggins 2000; see also the previous discussion on $\mathrm{R} \mathrm{Scl}$ and on mutiple winds). Therefore, by "present" mass-loss rate we refer to an average over the last $\sim 20-50$ years.

The use of a near-infrared index, [2.20-3.77], ensures that our estimates are relevant to the present mass-loss rates as defined above (Winters et al. 2000). In the context of the evaluation of the replenishment of the ISM it is important to use an estimate corresponding to a stage of the star for which it can be considered as stationary. Another advantage of this approach is that these relations are distance independent, so that an error on the distance estimation would not translate into an error on $\dot{M}$ (apart for the small interstellar correction on the color). On the other hand the correlations which have been found apply to mean color indices averaged over several periods. From Le Bertre (1997) we evaluate to a factor 2 the error made by using indices based on single epoch measurements such as those acquired by the IRTS. Therefore the individual estimates of $\dot{M}$ should be handled with care.

\subsection{Results}

The results of the present study are given in Table 4 . They concern the 126 objects that passed all our selection criteria: detection by the MIRS of a compact stellar source without PAH-emission, association to an IRAS source, colors typical of a mass-losing AGB star and a NIRS spectrum typical of an $M, C$ or S-type star. Each source is refered to with an index that we use throughout the present work and by its IRAS name. We give the dereddened [2.20] magnitude and [2.20-3.77] color, the estimated distance from the Sun, $d_{\text {Sun }}$, the distance above the galactic plane, $z$, the distance to the Galactic Centre, $d_{\mathrm{GC}}$, the mass-loss rate, $\dot{M}$, estimated using the relations given in Sect. 3.4 and finally a label defining the chemical composition of the circumstellar shell ( $\mathrm{C}$ for carbon-rich, $\mathrm{O}$ for oxygen-rich). The 16 sources observed at $3 \mathrm{kbps}$ are reported separately at the end of the table.

\section{Discussion}

\subsection{Contamination}

As we have checked individually every spectrum of the 130 candidates, we can be confident that the 126 sources reported in Table 4 are bona-fide AGB stars. For this selection, only 2 sources were misclassified with the Epchtein et al. (1987) criterion and this has been corrected for the present work.
Nevertheless, for future works, it is important to evaluate the contamination of the region defined in Sect. 2.5 by other sources which are not genuine mass-losing AGB stars. In the sample of 130 stars found in this region after dereddening, only 4 are not unambiguously related to the AGB; this includes the special cases of GM CMa, a carbon star, and of TW Cam, an RV Tauri star. It thus seems that the contamination is at a level $<5 \%$. It is noteworthy that the 10 stars in Table 1 are all late-type giants.

On the other hand, it is possible that many early-type stars have been removed from our initial sample together with the HII regions (see Sect. 2.3). Another bias that could have affected early-type stars arises from the difficulty of extracting NIRS sources close to the Galactic Plane, because of the large beam. Therefore we could have underestimated the contamination by this type of source. This discussion illustrates the importance of spectrophotometric data in the $1-5 \mu \mathrm{m}$ region which allows to pin down early-type stars.

\subsection{Space distribution of the sources}

In Fig. 6, we present the projection of the final sample on the Galactic Plane (GP). One notes a clear preference for carbon stars to be located outside the solar circle and the opposite, for oxygen-rich sources. At more than $10 \mathrm{kpc}$ from the Galactic Centre, we have 17 carbon stars and 3 oxygen-rich ones. Between 8 and $10 \mathrm{kpc}$, we get respectively 17 and 23 sources. Finally at less than $8 \mathrm{kpc}$, we get 6 carbon sources and 60 oxygen-rich ones. The two populations seem to balance at about the distance of the Sun to the Galactic Centre, in agreement with Jura \& Kleinmann (1989). In previous studies (Jura 1991; Guglielmo et al. 1998), it has been found that the surface density of carbon sources is almost constant within $3 \mathrm{kpc}$ of the Sun. With the present selection of 40 sources, we cannot confirm this distribution, but we should be able to revisit it on the basis of the complete NIRS data set which should provide $10-15$ times more sources.

In the $Z$ direction, most of the sources (74) are within $200 \mathrm{pc}$ of the GP, 32 are found between 200 and $400 \mathrm{pc}$, and 20 , beyond $400 \mathrm{pc}$. The carbon-rich sources tend to be more concentrated towards the GP, but in view of the present size of the sample this effect is only marginal.

\subsection{Contribution to the replenishment of the ISM}

Our ultimate goal is to quantify the contributions of the 2 different kinds of mass-losing AGB stars to the replenishment of the ISM at different distances from the Galactic Centre and different distances from the GP. Our present selection of 126 AGB sources suffers from many biases coming from the extraction of NIRS sources. Nevertheless, for a first step, we assume that these biases affect equally the carbon-rich and the oxygen-rich sources.

In Table 5, we sum up the contributions of our sources in 3 belts. As our sample is not complete the absolute 
Table 4. Properties of the 126 selected mass-losing AGB sources. The magnitudes and colors are corrected for reddenning. (C) stands for a carbon-rich composition and (O), for an oxygen-rich composition.

\begin{tabular}{|c|c|c|c|c|c|c|c|c|}
\hline Index & IRAS name & {$[2.20]$} & {$[2.20-3.77]$} & $\begin{array}{c}d_{\text {Sun }} \\
\text { pc }\end{array}$ & $\begin{array}{c}z \\
\mathrm{pc}\end{array}$ & $\begin{array}{l}d_{\mathrm{GC}} \\
\mathrm{kpc}\end{array}$ & $\begin{array}{c}\log \dot{M}_{-6} \\
10^{-6} M_{\odot} \mathrm{yr}^{-1}\end{array}$ & composition \\
\hline 793 & $01159+7220$ & 1.60 & 1.19 & 729. & 125 . & 8.93 & -0.063 & $\mathrm{O}$ \\
\hline 790 & $01411+7104$ & 4.03 & 1.16 & 2223 . & 345 . & 9.98 & -0.760 & $\mathrm{C}$ \\
\hline 775 & $02596+6639$ & 4.36 & 2.13 & 2529 . & 321. & 10.44 & 0.199 & $\mathrm{C}$ \\
\hline 749 & $03374+6229$ & 0.43 & 0.79 & 424. & 44. & 8.83 & -1.366 & $\mathrm{C}$ \\
\hline 721 & $03575+5922$ & 4.03 & 1.27 & 2222 . & 197. & 10.39 & -0.616 & $\mathrm{C}$ \\
\hline 708 & $04207+5548$ & 3.84 & 0.88 & 2038. & 162. & 10.31 & -1.206 & $\mathrm{C}$ \\
\hline 684 & $04449+4951$ & 4.49 & 2.57 & 1939. & 111. & 10.31 & 0.483 & $\mathrm{C}$ \\
\hline 683 & $04504+4949$ & 2.70 & 1.03 & 1205 . & 82. & 9.62 & -0.946 & $\mathrm{C}$ \\
\hline 159 & $05096-4834$ & 0.18 & 0.73 & 379. & -224 & 8.59 & -1.538 & $\mathrm{O}$ \\
\hline 664 & $05106+4520$ & 5.95 & 2.65 & 3587. & 244. & 11.97 & 0.526 & $\mathrm{C}$ \\
\hline 627 & $05149+3511$ & 3.77 & 1.84 & 1974. & -50 & 10.46 & -0.028 & $\mathrm{C}$ \\
\hline 616 & $05185+3227$ & 1.89 & 0.95 & 829. & -34 & 9.32 & -1.076 & $\mathrm{C}$ \\
\hline 617 & $05204+3227$ & 5.28 & 1.54 & 3959. & -141 & 12.44 & 0.467 & $\mathrm{O}$ \\
\hline 648 & $05214+4001$ & 4.00 & 1.26 & 2199. & 93. & 10.66 & -0.639 & $\mathrm{C}$ \\
\hline 623 & $05238+3406$ & 1.35 & 1.51 & 647. & -6 & 9.14 & -0.345 & $\mathrm{C}$ \\
\hline 577 & $05325+2351$ & 2.76 & 0.89 & 1243. & -100 & 9.74 & -0.825 & $\mathrm{O}$ \\
\hline 606 & $05345+3002$ & 3.20 & 0.95 & 1522. & -23 & 10.02 & -1.081 & $\mathrm{C}$ \\
\hline 190 & $05345-4406$ & 2.46 & 0.74 & 1082. & -566 & 8.86 & -1.493 & $\mathrm{O}$ \\
\hline 573 & $05352+2247$ & 3.00 & 0.92 & 1384. & -112 & 9.88 & -1.138 & $\mathrm{C}$ \\
\hline 175 & $05418-4628$ & 1.02 & 0.85 & 557. & -284 & 8.65 & -1.254 & $\mathrm{C}$ \\
\hline 583 & $05421+2424$ & 1.50 & 0.82 & 694. & -29 & 9.19 & -1.305 & $\mathrm{C}$ \\
\hline 562 & $05426+2040$ & 0.32 & 0.78 & 403. & -30 & 8.90 & -1.375 & $\mathrm{C}$ \\
\hline 575 & $05465+2321$ & 4.24 & 0.96 & 2449 . & -89 & 10.94 & -1.055 & $\mathrm{C}$ \\
\hline 184 & $05510-4522$ & 3.33 & 0.79 & 1615. & -781 & 9.04 & -1.244 & $\mathrm{O}$ \\
\hline 484 & $05591+0638$ & 4.26 & 1.03 & 2479 . & -342 & 10.82 & -0.428 & $\mathrm{O}$ \\
\hline 463 & $06026+0322$ & 6.38 & 2.11 & 6506. & -992 & 14.59 & 0.188 & $\mathrm{C}$ \\
\hline 397 & $06266-1148$ & 3.40 & 0.96 & 1663. & -297 & 9.79 & -0.618 & $\mathrm{O}$ \\
\hline 341 & $06496-1858$ & 1.76 & 0.85 & 781. & -115 & 9.02 & -1.004 & $\mathrm{O}$ \\
\hline 340 & $06550-1915$ & 3.87 & 0.88 & 2070. & -270 & 9.92 & -0.879 & $\mathrm{O}$ \\
\hline 320 & $06588-2138$ & 5.41 & 1.89 & 4196. & -569 & 11.48 & 0.011 & $\mathrm{C}$ \\
\hline 354 & 07019-1631 & 3.03 & 0.76 & 1407. & -117 & 9.48 & -1.344 & $\mathrm{O}$ \\
\hline 368 & $07028-1456$ & 4.29 & 2.46 & 1921. & -129 . & 9.89 & 0.417 & $\mathrm{C}$ \\
\hline 359 & $07031-1604$ & 4.91 & 1.29 & 3336. & -252 & 10.98 & -0.595 & $\mathrm{C}$ \\
\hline 405 & $07118-1022$ & 4.96 & 1.89 & 3412. & 12. & 11.18 & 0.012 & $\mathrm{C}$ \\
\hline 371 & $07145-1428$ & 4.26 & 1.64 & 2470 . & -49 & 10.30 & -0.208 & $\mathrm{C}$ \\
\hline 383 & $07180-1314$ & 3.53 & 1.41 & 1766. & 7. & 9.77 & 0.297 & $\mathrm{O}$ \\
\hline 370 & $07197-1451$ & 2.54 & 0.72 & 1122 . & -3 & 9.27 & -1.553 & $\mathrm{O}$ \\
\hline 375 & $07208-1410$ & 3.58 & 0.85 & 1808. & 11. & 9.78 & -1.243 & $\mathrm{C}$ \\
\hline 431 & $07227-0537$ & 5.45 & 1.62 & 4290. & 361. & 12.03 & -0.232 & $\mathrm{C}$ \\
\hline 429 & $07232-0544$ & 1.73 & 0.70 & 772. & 66. & 9.09 & -1.656 & $\mathrm{O}$ \\
\hline 445 & $07487-0229$ & 2.95 & 1.68 & 1355. & 282. & 9.52 & -0.171 & $\mathrm{C}$ \\
\hline 517 & $08138+1152$ & -0.67 & 0.74 & 256. & 104. & 8.70 & -1.478 & $\mathrm{O}$ \\
\hline 526 & $08186+1409$ & 3.18 & 0.72 & 1504. & 663. & 9.69 & -1.583 & $\mathrm{O}$ \\
\hline 9 & $11318-7256$ & 1.17 & 1.83 & 596. & -116 & 8.25 & -0.035 & $\mathrm{C}$ \\
\hline 773 & $12544+6615$ & 0.15 & 0.85 & 374. & 291. & 8.63 & -1.254 & $\mathrm{C}$ \\
\hline 12 & $13350-7221$ & 2.86 & 0.87 & 1297. & -228 & 7.81 & -0.908 & $\mathrm{O}$ \\
\hline 13 & $13417-7221$ & 4.08 & 0.80 & 2278 . & -404 & 7.37 & -1.179 & $\mathrm{O}$ \\
\hline 29 & $14188-6943$ & 2.69 & 0.71 & 1202. & -179 . & 7.78 & -1.642 & $\mathrm{O}$ \\
\hline 36 & $14473-6842$ & 3.80 & 0.79 & 1999. & -300 & 7.28 & -1.236 & $\mathrm{O}$ \\
\hline 54 & $15155-6531$ & 3.34 & 1.16 & 1622. & -201 & 7.39 & -0.130 & $\mathrm{O}$ \\
\hline 60 & $15332-6430$ & 1.96 & 1.18 & 859. & -110 & 7.87 & -0.078 & $\mathrm{O}$ \\
\hline 55 & $15389-6517$ & 3.09 & 0.71 & 1442. & -209 & 7.47 & -1.627 & $\mathrm{O}$ \\
\hline 116 & $16156-5613$ & 2.99 & 1.12 & 1379 . & -106 & 7.36 & -0.213 & $\mathrm{O}$ \\
\hline 146 & $16376-5040$ & 2.92 & 0.74 & 1335. & -67 & 7.31 & -1.473 & $\mathrm{O}$ \\
\hline 132 & $16410-5240$ & 3.22 & 0.87 & 1531. & -124 . & 7.16 & -0.912 & $\mathrm{O}$ \\
\hline
\end{tabular}


Table 4. continued.

\begin{tabular}{|c|c|c|c|c|c|c|c|c|}
\hline Index & IRAS name & {$[2.20]$} & {$[2.20-3.77]$} & $\begin{array}{c}d_{\text {Sun }} \\
\text { pc }\end{array}$ & $\begin{array}{c}z \\
\mathrm{pc}\end{array}$ & $\begin{array}{l}d_{\mathrm{GC}} \\
\mathrm{kpc}\end{array}$ & $\begin{array}{c}\log \dot{M}_{-6} \\
10^{-6} M_{\odot} \mathrm{yr}^{-1}\end{array}$ & composition \\
\hline 142 & $16455-5124$ & 3.71 & 0.74 & 1924. & -147. & 6.80 & -1.449 & $\mathrm{O}$ \\
\hline 178 & $16507-4644$ & 4.07 & 0.99 & 2269. & -80 & 6.42 & -0.523 & $\mathrm{O}$ \\
\hline 168 & $16538-4652$ & 2.36 & 0.81 & 1034. & -45 . & 7.54 & -1.137 & $\mathrm{O}$ \\
\hline 182 & $16558-4526$ & 2.42 & 0.76 & 1061. & -34 & 7.50 & -1.350 & $\mathrm{O}$ \\
\hline 207 & $16598-4117$ & 2.05 & 1.06 & 896. & 1. & 7.64 & -0.345 & $\mathrm{O}$ \\
\hline 195 & $17054-4342$ & 3.02 & 1.02 & 1397. & -53 & 7.17 & -0.966 & $\mathrm{C}$ \\
\hline 227 & $17163-3835$ & 0.90 & 0.86 & 527. & -7 & 7.98 & -0.931 & $\mathrm{O}$ \\
\hline 234 & $17187-3750$ & 1.33 & 0.94 & 641. & -8 & 7.87 & -0.684 & $\mathrm{O}$ \\
\hline 252 & $17209-3318$ & 4.15 & 2.52 & 1720. & 43. & 6.79 & 0.452 & $\mathrm{C}$ \\
\hline 263 & $17209-3126$ & 3.52 & 1.45 & 1762 . & 75 . & 6.75 & 0.349 & $\mathrm{O}$ \\
\hline 230 & $17244-3827$ & 3.16 & 0.73 & 1490. & -55 & 7.04 & -1.541 & $\mathrm{O}$ \\
\hline 251 & $17328-3327$ & 0.60 & 0.92 & 458. & -6 & 8.04 & -0.729 & $\mathrm{O}$ \\
\hline 308 & $17346-2312$ & 3.36 & 0.94 & 1635. & 128. & 6.88 & -0.682 & $\mathrm{O}$ \\
\hline 292 & $17346-2612$ & 4.34 & 0.85 & 2571. & 128. & 5.93 & -0.990 & $\mathrm{O}$ \\
\hline 307 & $17367-2319$ & 2.62 & 1.12 & 1162. & 82. & 7.34 & -0.213 & $\mathrm{O}$ \\
\hline 326 & $17389-2045$ & 2.26 & 1.02 & 987. & 86. & 7.52 & -0.440 & $\mathrm{O}$ \\
\hline 366 & $17462-1511$ & 3.69 & 0.97 & 1905. & 211. & 6.66 & -0.596 & $\mathrm{O}$ \\
\hline 365 & $17468-1503$ & 4.53 & 1.01 & 2801. & 306. & 5.81 & -0.460 & $\mathrm{O}$ \\
\hline 362 & $17482-1529$ & 2.93 & 0.82 & 1339. & 134. & 7.20 & -1.111 & $\mathrm{O}$ \\
\hline 376 & $17541-1404$ & 2.79 & 0.76 & 1260 . & 117. & 7.29 & -1.361 & $\mathrm{O}$ \\
\hline 425 & $17573-0807$ & 2.75 & 0.99 & 1233. & 161. & 7.36 & -0.524 & $\mathrm{O}$ \\
\hline 426 & $18039-0813$ & 1.63 & 1.02 & 738. & 78. & 7.82 & -0.458 & $\mathrm{O}$ \\
\hline 427 & $18054-0649$ & 3.80 & 0.80 & 2001. & 222 . & 6.70 & -1.169 & $\mathrm{O}$ \\
\hline 626 & $18076+3445$ & 3.81 & 1.55 & 2008. & 789. & 7.79 & 0.477 & $\mathrm{O}$ \\
\hline 485 & $18202+0636$ & 4.17 & 0.86 & 2375 . & 385. & 6.74 & -0.962 & $\mathrm{O}$ \\
\hline 504 & $18209+0928$ & 4.09 & 0.98 & 2290 . & 418. & 6.88 & -0.552 & $\mathrm{O}$ \\
\hline 637 & $18306+3657$ & 0.21 & 0.84 & 383. & 128. & 8.36 & -1.265 & $\mathrm{C}$ \\
\hline 568 & $18444+2143$ & 3.44 & 0.71 & 1700 . & 316. & 7.59 & -1.609 & $\mathrm{O}$ \\
\hline 559 & $18512+2029$ & 4.49 & 1.59 & 2754 . & 422. & 7.14 & 0.520 & $\mathrm{O}$ \\
\hline 538 & $18561+1642$ & 2.57 & 0.81 & 1137. & 119. & 7.80 & -1.162 & $\mathrm{O}$ \\
\hline 574 & $19029+2305$ & 4.35 & 0.96 & 2583. & 337. & 7.34 & -0.620 & $\mathrm{O}$ \\
\hline 545 & $19090+1746$ & 3.23 & 1.00 & 1540 . & 103. & 7.63 & -0.513 & $\mathrm{O}$ \\
\hline 528 & $19111+1404$ & 2.86 & 0.89 & 1296. & 38. & 7.69 & -0.846 & $\mathrm{O}$ \\
\hline 654 & $19158+4243$ & 3.45 & 0.74 & 1702 . & 401. & 8.21 & -1.471 & $\mathrm{O}$ \\
\hline 541 & $19172+1706$ & 2.28 & 0.73 & 996. & 31. & 7.92 & -1.536 & $\mathrm{O}$ \\
\hline 503 & $19192+0922$ & 3.42 & 2.36 & 1389. & -57 & 7.58 & 1.082 & $\mathrm{O}$ \\
\hline 496 & $19224+0732$ & 3.61 & 1.11 & 1834. & -124 & 7.28 & -0.234 & $\mathrm{O}$ \\
\hline 491 & $19248+0658$ & 2.97 & 1.95 & 1368. & -111 & 7.57 & 0.061 & $\mathrm{C}$ \\
\hline 514 & $19264+1132$ & 3.56 & 0.99 & 1790. & -90 & 7.41 & -0.523 & $\mathrm{O}$ \\
\hline 686 & $19354+5005$ & 0.47 & 0.75 & 433. & 103. & 8.46 & -1.399 & $\mathrm{O}$ \\
\hline 482 & $19354+0636$ & 3.30 & 0.94 & 1593. & -198 & 7.45 & -1.100 & $\mathrm{C}$ \\
\hline 465 & $19412+0337$ & 2.76 & 1.51 & 1241. & -213 & 7.64 & 0.425 & $\mathrm{O}$ \\
\hline 448 & $19433-0158$ & 4.93 & 1.63 & 3367. & -754 & 6.23 & 0.563 & $\mathrm{O}$ \\
\hline 466 & $19454+0355$ & 3.06 & 1.15 & 1427. & -264 & 7.54 & -0.138 & $\mathrm{O}$ \\
\hline 446 & $19550-0201$ & -0.01 & 0.91 & 347. & -93 & 8.24 & -0.780 & $\mathrm{O}$ \\
\hline 428 & $20077-0625$ & 2.34 & 2.31 & 876. & -306 & 7.85 & 1.057 & $\mathrm{O}$ \\
\hline 372 & $20084-1425$ & 2.91 & 1.30 & 1326. & -541 & 7.46 & -0.588 & $\mathrm{C}$ \\
\hline 750 & $20215+6243$ & 2.08 & 0.76 & 908. & 226. & 8.65 & -1.347 & $\mathrm{O}$ \\
\hline 377 & $20234-1357$ & 2.62 & 1.09 & 1164. & -532 & 7.63 & -0.285 & $\mathrm{O}$ \\
\hline 321 & $20296-2151$ & 2.65 & 0.76 & 1181. & -617 & 7.58 & -1.386 & $\mathrm{O}$ \\
\hline 225 & $21069-3843$ & 2.00 & 0.93 & 875. & -596 & 7.86 & -0.720 & $\mathrm{O}$ \\
\hline 212 & $21206-4054$ & 1.85 & 0.94 & 817. & -584 & 7.93 & -0.663 & $\mathrm{O}$ \\
\hline 785 & $21262+7000$ & 1.41 & 1.32 & 667. & 160. & 8.71 & -0.557 & $\mathrm{C}$ \\
\hline 169 & $21453-4708$ & 1.75 & 0.74 & 778. & -590 & 8.00 & -1.480 & $\mathrm{O}$ \\
\hline 791 & $22348+7149$ & 3.27 & 0.93 & 1566. & 322. & 9.20 & -0.711 & $\mathrm{O}$ \\
\hline
\end{tabular}


Table 4. continued, objects observed at $3 \mathrm{kbps}$.

\begin{tabular}{rcccccccc}
\hline Index & IRAS name & {$[2.20]$} & {$[2.20-3.77]$} & $\begin{array}{c}d_{\text {Sun }} \\
\mathrm{pc}\end{array}$ & $\begin{array}{c}z \\
\mathrm{pc}\end{array}$ & $\begin{array}{c}d_{\mathrm{GC}} \\
\mathrm{kpc}\end{array}$ & $\begin{array}{c}\log \dot{M}_{-6} \\
10^{-6} M_{\odot} \mathrm{yr}^{-1}\end{array}$ & composition \\
\hline 787 & $02588+6956$ & 3.55 & 0.79 & 1788. & 313. & 9.80 & -1.248 & $\mathrm{O}$ \\
754 & $03287+6249$ & 3.55 & 0.74 & 1782. & 176. & 9.93 & -1.487 & $\mathrm{O}$ \\
609 & $05334+3057$ & 4.87 & 1.56 & 3275. & -33. & 11.77 & -0.288 & $\mathrm{C}$ \\
411 & $06230-0930$ & 6.43 & 2.65 & 4455. & -784. & 12.24 & 0.526 & $\mathrm{C}$ \\
331 & $07098-2012$ & 3.92 & 2.88 & 1184. & -98. & 9.25 & 0.647 & $\mathrm{C}$ \\
189 & $07178-4429$ & 5.84 & 1.59 & 5126. & -1249. & 10.82 & 0.517 & $\mathrm{O}$ \\
21 & $10342-7027$ & 3.22 & 0.86 & 1533. & -286. & 8.05 & -0.961 & $\mathrm{O}$ \\
2 & $12593-7355$ & 4.73 & 0.95 & 3071. & -608. & 7.27 & -0.641 & $\mathrm{O}$ \\
24 & $13408-7021$ & 2.39 & 0.78 & 1046. & -150. & 7.91 & -1.384 & $\mathrm{C}$ \\
242 & $17107-3611$ & 3.42 & 0.79 & 1680. & 42. & 6.85 & -1.218 & $\mathrm{O}$ \\
258 & $17215-3237$ & 3.46 & 1.01 & 1711. & 52. & 6.80 & -0.469 & $\mathrm{O}$ \\
301 & $17380-2429$ & 3.92 & 0.91 & 2118. & 113. & 6.39 & -0.759 & $\mathrm{O}$ \\
339 & $17540-1919$ & 2.17 & 1.19 & 947. & 45. & 7.57 & -0.059 & $\mathrm{O}$ \\
432 & $18050-0518$ & 4.02 & 1.28 & 2213. & 278. & 6.54 & 0.102 & $\mathrm{O}$ \\
472 & $18125+0447$ & 3.79 & 0.83 & 1994. & 356. & 6.94 & -1.046 & $\mathrm{O}$ \\
489 & $19426+0650$ & 3.34 & 0.85 & 1620. & -243. & 7.46 & -0.968 & $\mathrm{O}$ \\
\hline
\end{tabular}

values have no immediate meaning. Also, it appears that the contributions from the sources with largest mass loss rates dominate these sums which therefore are strongly affected by the small number statistics (see below, and Table 6). In spite of that, it is clear that the oxygenrich sources dominate the replenishment of the ISM by AGB stars for $d_{\mathrm{GC}}<8 \mathrm{kpc}$, by a factor $10-12$, and the carbon-rich sources, for $d_{\mathrm{GC}}>10 \mathrm{kpc}$, by a factor $3-4$. It means that the composition of the ISM, especially in its dust component, should vary considerably between $\sim 6$ and $12 \mathrm{kpc}$ from the Galactic Centre.

In Table 6, we sum up the contributions of our 126 sources in mass-loss-rate bins. The 20 sources with $\dot{M}$ in the range $10^{-6}-10^{-5} M_{\odot} \mathrm{yr}^{-1}$ contribute about as much as the rest of the sample to the replenishment of the ISM. Two objects with $\dot{M}>10^{-5} M_{\odot} \mathrm{yr}^{-1}$ contribute $\sim 1 / 4$ of the total from all the sources. These two sources (503 and 428) are found to have mass-loss rates $\sim 1.2 \times 10^{-5} M_{\odot} \mathrm{yr}^{-1}$. Our sample is limited in size and we do not get AGB mass losing sources with larger rates, although such sources are known to exist with rates up to $10^{-4} M_{\odot} \mathrm{yr}^{-1}$ (Habing 1996). It would be important to evaluate their contribution because, although they seem to be extremely rare, they might still contribute substantially to the replenishment of the ISM.

From these remarks, it becomes clear that a proper determination of the replenishment of the ISM close to the Sun requires the exploration of a large volume inside a torus centered on the Galactic Centre and enclosing the Sun, in order to overcome the effect of small number statistics.

\section{Conclusion}

We have shown that the data acquired by the NearInfrared Spectrometer of the IRTS can be used to obtain an estimate of the contribution of carbon-rich and
Table 5. Numbers of carbon-rich and oxygen-rich sources and their respective contributions to the replenishment of the ISM at different distances from the Galactic Centre.

\begin{tabular}{|r|rr|rr|}
\hline$d_{\mathrm{GC}}$ & C-rich & & O-rich & \\
& $N$ & $\sum \dot{M}_{-6}$ & $N$ & $\sum \dot{M}_{-6}$ \\
& & & & \\
\hline $6-8 \mathrm{kpc}$ & 6 & 4.47 & 58 & 51.46 \\
$8-10 \mathrm{kpc}$ & 17 & 10.18 & 23 & 4.60 \\
$10-12 \mathrm{kpc}$ & 14 & 13.06 & 2 & 3.66 \\
\hline
\end{tabular}

Table 6. Numbers of carbon-rich and oxygen-rich sources and their respective contributions to the replenishment of the ISM for different mass-loss rates.

\begin{tabular}{|r|rr|rr|}
\hline$\dot{M}$ & C-rich & & O-rich & \\
$\left(M_{\odot} \mathrm{yr}^{-1}\right)$ & $N$ & $\sum \dot{M}_{-6}$ & $N$ & $\sum \dot{M}_{-6}$ \\
\hline $2 \times 10^{-8}-10^{-7}$ & 14 & 0.87 & 33 & 1.50 \\
$10^{-7}-10^{-6}$ & 15 & 6.36 & 42 & 13.80 \\
$10^{-6}-10^{-5}$ & 11 & 25.96 & 9 & 24.33 \\
$10^{-5}-10^{-4}$ & 0 & & 2 & 23.48 \\
\hline
\end{tabular}

oxygen-rich mass-losing AGB stars to the replenishment of the ISM at different distances $(\leq 3 \mathrm{kpc})$ from the Sun. The combination with IRAS data helps to sort out the sources. This approach will be developed in Paper II on the basis of the complete NIRS data.

We note that an experiment similar to the NIRS/IRTS with a smaller entrance aperture and a better sensitivity could allow to easily extend such a study to the whole Galaxy. For sources which have no good IRAS fluxes at 12 and $25 \mu \mathrm{m}$, low-resolution spectroscopy $(R \sim 50)$ in the $1-5 \mu \mathrm{m}$ range may as well be used to discriminate O-rich from C-rich stars in most situations. Also, this kind of 


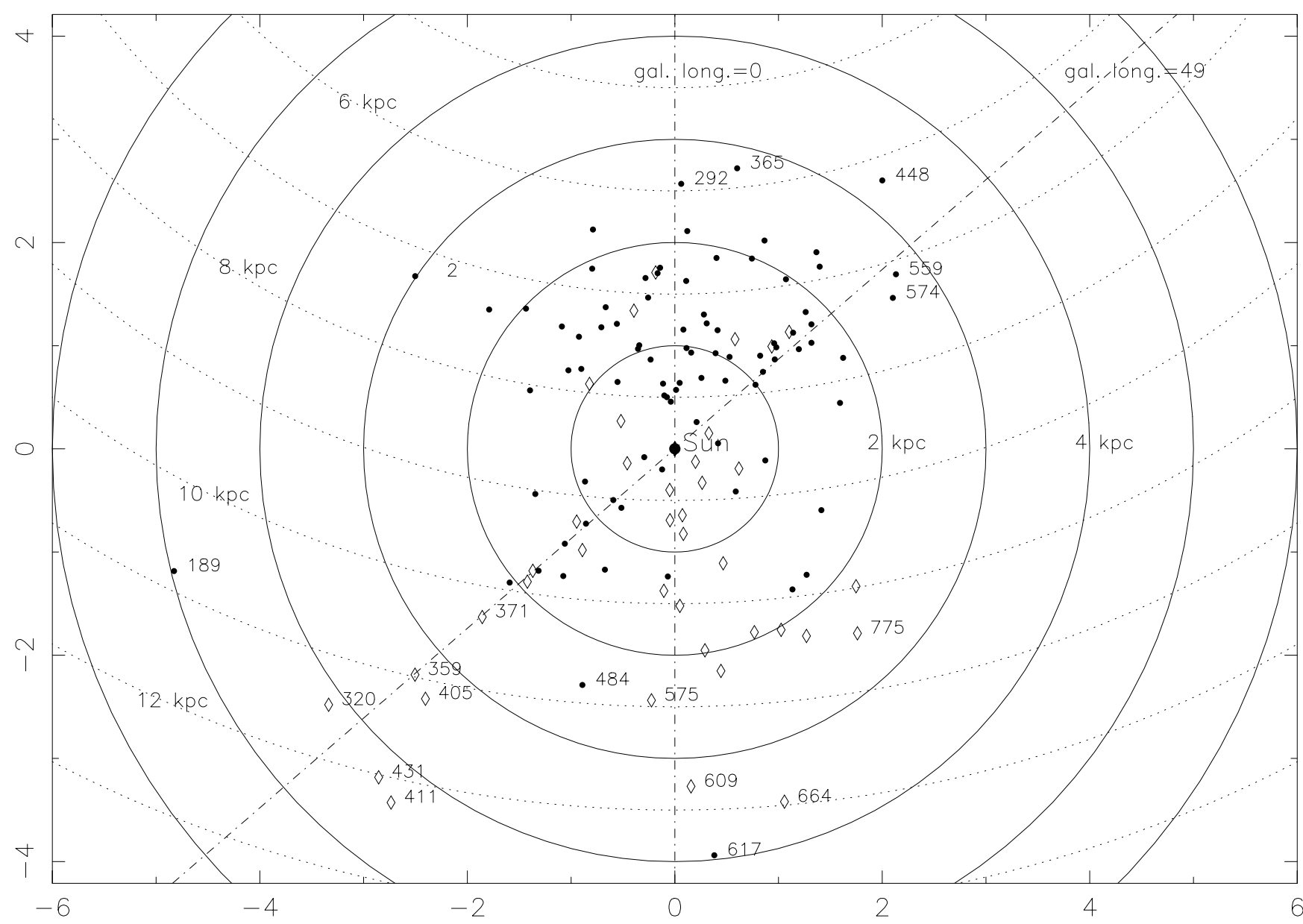

Fig. 6. Projection on the Galactic Plane of the final sample (126 sources). The carbon stars are represented with diamonds $(\diamond)$ and the oxygen-rich stars with dots $(\bullet)$. The dotted lines are circles centered on the Galactic Centre.

spectral data is useful to identify contaminating and misclassified sources.

Acknowledgements. T. Le Bertre is grateful to the Institute of Space and Astronautical Science (ISAS) for an invitation to stay in Japan and for the permission to work on IRTS data. J. M. Winters acknowledges financial support by the CNRS. This research has made use of the SIMBAD database, operated at CDS, Strasbourg, France. This work has been supported by the Paris Observatory through the fund BQR 2000. We gratefully acknowledge useful comments from A. Jorissen and K. Justtanont.

\section{Appendix A: Bolometric corrections}

To estimate the bolometric corrections for O-rich sources we used the sample of infrared sources observed by Le Bertre (1993) whose energy distributions have been modelled by Le Sidaner \& Le Bertre (1996). We adopted the average $K-L^{\prime}$ index from the observations, and the average $\mathrm{m}_{\text {bol }}$ from the modelling. The $\mathrm{OH} / \mathrm{IR}$ sources were excluded because their bolometric magnitudes might be affected by the correction for interstellar extinction which is uncertain at long wavelengths. For the C-rich sources we used the sample of Le Bertre (1992) with the modelling of

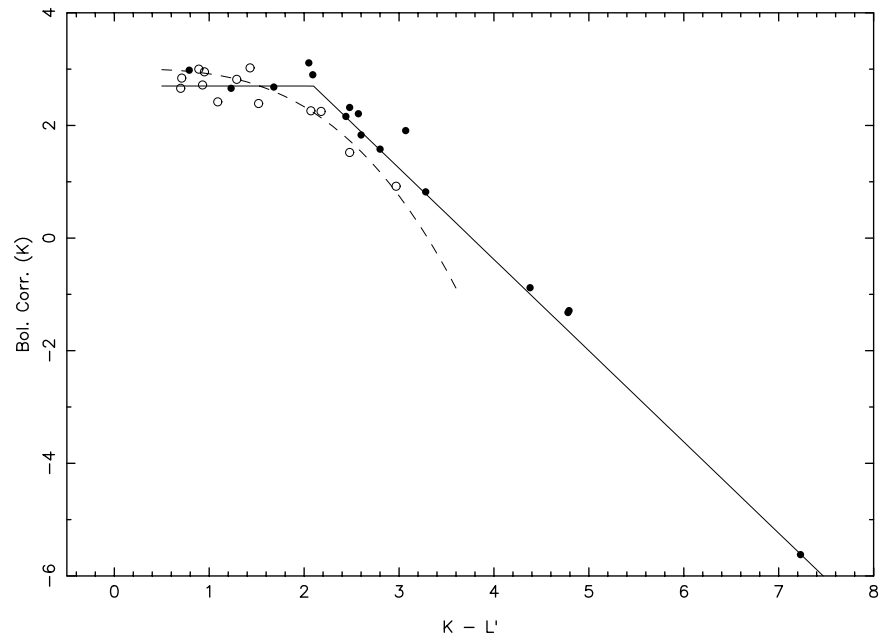

Fig. A.1. $B C_{K}$ versus $K-L^{\prime}$ for the samples of O-rich (circles) and C-rich (dots) sources discussed in the Appendix. Solid line: adopted relation. Dashed line: van Loon et al. (1999) relation.

Le Bertre (1997). We excluded AFGL 971 whose spectrum is affected by interstellar extinction. 


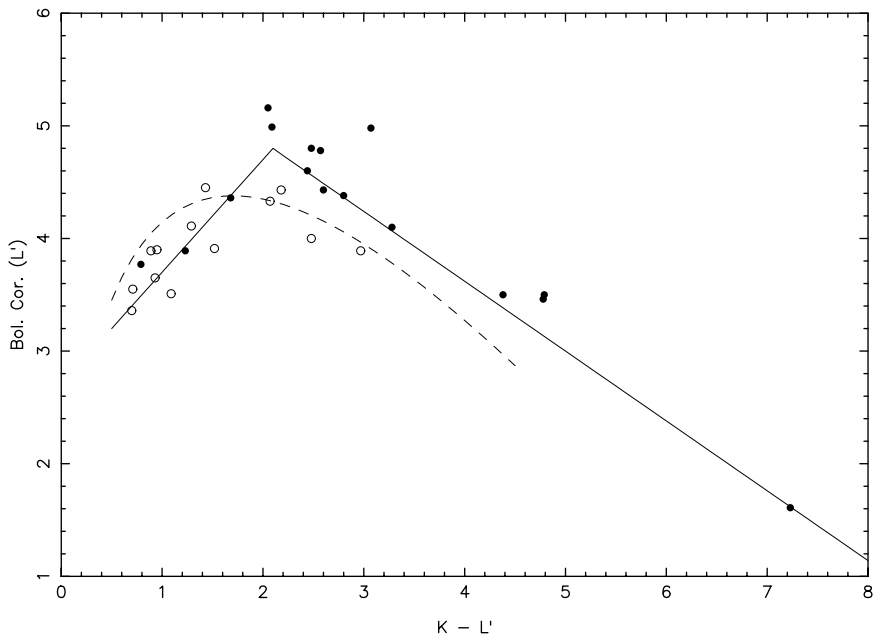

Fig. A.2. $B C_{L^{\prime}}$ versus $K-L^{\prime}$ for the samples of O-rich (circles) and C-rich (dots) sources discussed in the Appendix. Solid line: recommended relation. Dashed line: Wood et al. (1998) relation.

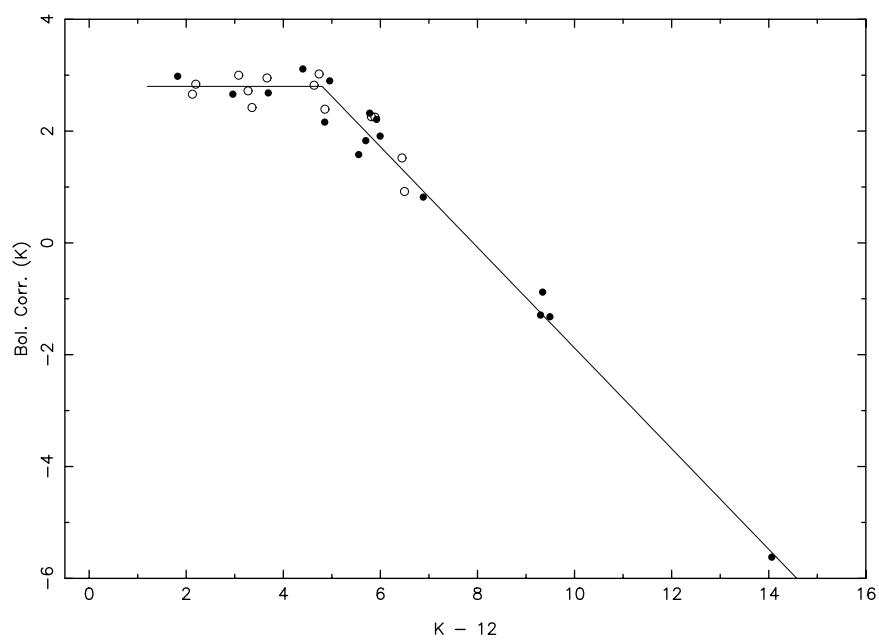

Fig. A.3. $B C_{K}$ versus $K-[12]$ for the samples of O-rich (circles) and C-rich (dots) sources discussed in the Appendix. Solid line: recommended relation.

The results are presented in Fig. A.1. A similar work has been performed by van Loon et al. (1999) who obtained comparable results. They find that there is virtually no difference between C-rich and O-rich sources. The fit to all their data is shown as a dashed line $\left(K-L^{\prime} \leq 3.6\right)$. From our samples it is not clear if there is a difference between the corrections for the 2 kinds of sources. We have 4 (carbon-rich) sources with $K-L^{\prime} \geq 3.6$ which deviate from an extrapolation of the van Loon et al. relation. Therefore, we adopt the relations (common to $\mathrm{O}$ and $\mathrm{C}$ rich sources):

$B C_{K}=2.7$,

for $\left(K-L^{\prime}\right) \leq 2.1$, and

$B C_{K}=6.1-1.62 \times\left(K-L^{\prime}\right)$,

for $2.1 \leq\left(K-L^{\prime}\right) \leq 7$.

Instead of evaluating the bolometric correction with respect to $K$, we can evaluate it with respect to $L^{\prime}$. This approach has also been followed by Wood et al. (1998), but they used $L(3.59 \mu \mathrm{m})$ instead of $L^{\prime}(3.79 \mu \mathrm{m})$ so that a direct comparison is not feasible. Our data can be represented with Fig. A.2:

$B C_{L^{\prime}}=2.7+\left(K-L^{\prime}\right)$

for $\left(K-L^{\prime}\right) \leq 2.1$, and

$B C_{L^{\prime}}=6.10-0.62 \times\left(K-L^{\prime}\right)$,

for $\left(K-L^{\prime}\right) \geq 2.1$.

Another index which can be useful to consider is $K-$ [12], where [12] is the IRAS $12 \mu \mathrm{m}$ magnitude. The results are presented in Fig. A.3. The data are well represented by the relations:

$B C_{K}=2.8$,

for $(K-[12]) \leq 4.8$, and

$B C_{K}=7.12-0.9 \times(K-[12])$,

for $(K-[12]) \geq 4.8$.

\section{References}

Alvarez, R., Lançon, A., Plez, B., \& Wood, P. R. 2000, A\&A, 353,322

Cohen, M., Walker, R. G., Carter, B., et al. 1999, AJ, 117, 1864

Epchtein, N. 1999, Asymptotic Giant Branch Stars, IAU Symp., 191, 97

Epchtein, N., Le Bertre, T., Lépine, J. R. D., et al. 1987, A\&AS, 71, 39

Fleischer, A. J., Gauger, A., \& Sedlmayr, E. 1995, A\&A, 297, 543

Fouqué, P., Le Bertre, T., Epchtein, N., Guglielmo, F., \& Kerschbaum, F. 1992, A\&AS, 93, 151

Gehrz, R. D. 1989, Interstellar Dust, IAU Symp., 135, 445

Guglielmo, F., Le Bertre, T., Epchtein, N. 1998, A\&A, 334, 609

Habing, H. J. 1996, A\&AR, 7, 97

Jones, T. J., Ashley, M., Hyland, A. R., \& Ruelas-Mayorga, A. 1981, MNRAS, 197, 413

Jones, T. W., \& Merrill, K. M. 1976, ApJ, 209, 509

Jura, M. 1987, ApJ, 313, 743

Jura, M. 1991, A\&AR, 2, 227

Jura, M., \& Kleinmann, S. G. 1989, ApJ, 341, 359

Kahane, C., \& Jura, M. 1996, A\&A, 310, 952

Knapp, G. R., \& Morris, M. 1985, ApJ, 292, 640

Knapp, G. R., Young, K., Lee, E., \& Jorissen, A. 1998, ApJS, 117, 209

Lafon, J.-P. J., \& Berruyer, N. 1991, A\&AR, 2, 249

Lançon, A., \& Wood, P. R. 2000, A\&AS, 146, 217

Le Bertre, T. 1992, A\&AS, 94, 377

Le Bertre, T. 1993, A\&AS, 97, 729

Le Bertre, T. 1997, A\&A, 324, 1059

Le Bertre, T., Epchtein, N., Guglielmo, F., \& Le Sidaner, P. 1994, Ap\&SS, 217, 105

Le Bertre, T., \& Winters, J. M. 1998, A\&A, 334, 173

Le Sidaner, P., \& Le Bertre, T. 1996, A\&A, 314, 896

Lloyd-Evans, T. 1997, MNRAS, 286, 839

Loup, C., Forveille, T., Omont, A., \& Paul, J. F. 1993, A\&AS, 99, 291

Massey, P., DeGioia-Eastwood, K., \& Waterhouse, E. 2001, AJ, 121,1050

Matsuura, M., Yamamura, I., Murakami, H., Freund, M. M., \& Tanaka, M. 1999, A\&A, 348, 579 
Matsuura, M., Yamamura, I., Murakami, H., \& Onaka, T. 2001, Post-AGB objects (proto-planetary nebulae) as a phase of stellar evolution, ed. R. Szczerba, \& S. K. Gorny, in press Mauron, N., \& Huggins, P. J. 2000, A\&A, 359, 707

Moshir, M., McCallon, H., Evans, T., et al. 1997, ASP Conf. Ser., 124, 21

Murakami, H., Freund, M. M., Ganga, K., et al. 1996, PASJ, 48, L41

Murakami, H., Matsuura, M., Cohen, M., et al. 2001, The calibration legacy of the ISO Mission, ed. L. Metcalfe, \& M. Kessler, ESA-SP 481, in press

Noda, M., Matsumoto, T., Murakami, H., et al. 1996, SPIE, 2817,248

Okuda, H., Matsumoto, T., Roellig, T. L. (eds.) 1997, Diffuse Infrared Radiation and the IRTS, ASP Conf. Ser., 124

Onaka, T., Ishihara, D., Ootsubo, T., et al. 2001, The calibration legacy of the ISO Mission, ed. L. Metcalfe, \& M. Kessler, ESA-SP 481, in press

Onaka, T., Yamamura, I., Tanabé, T., Roellig, T. L., Yuen, L. 1996, PASJ, 48, L59

Piccirillo, J. 1980, MNRAS, 190, 441
Rowan-Robinson, M., Lock, T. D., Walker, D. W., \& Harris, S. 1986, MNRAS, 222, 273

Sedlmayr, E. 1994, IAU Colloq. 146, Molecules in the Stellar Environment, ed. U. G. Jørgensen (Springer), 163

van der Veen, W. E. C. J., \& Habing, H. J. 1988, A\&A, 194, 125

van Loon, J. T., Groenewegen, M. A. T., de Koter, A., et al. 1999, A\&A, 351, 559

Wainscoat, R. J., Cohen, M., Volk, K., Walker, H. J., \& Schwartz, D. E. 1992, AJ, 83, 111

Winters, J. M., Le Bertre, T., Jeong, K. S., Helling, C., \& Sedlmayr, E. 2000, A\&A, 361, 641

Wood, P. R., Habing, H. J., \& McGregor, P. J. 1998, A\&A, 336,925

Yamamura, I., \& IRTS Team 1997, ASP Conf. Ser., 124, 72

Yamamura, I., \& de Jong, T. 2000, ISO beyond the peaks, ed. A. Salama, M. F. Kessler, K. Leech, \& B. Schultz, ESASP 456, 155

Yamamura, I., de Jong, T., Onaka, T., Cami, J., \& Waters, L. B. F. M. 1999, A\&A, 341, L9 